\title{
COMMON FIXED-POINTS FOR EQUICONTINUOUS SEMIGROUPS OF MAPPINGS
}

\author{
THEODORE MITCHELL ${ }^{1}$
}

\begin{abstract}
Let $S$ be a semigroup of equicontinuous self maps of $X$, a compact Hausdorff space. It is shown that if $S$ is left reversible (that is every pair of right ideals of $S$ has nonempty intersection), then there is a compact group $G$ of homeomorphisms of a retract $Y$ of $X$ with the property that $S$ has a common fixed-point in $X$ if and only if $G$ has a common fixed-point in $Y$. As an application, it is proved that if $F$ is a family of continuous commuting self maps of the closed unit interval $I$ with the property that for each $f \in F$, with one possible exception, the set of all iterates of $f$ is equicontinuous, then $I$ contains a common fixed-point of $F$.
\end{abstract}

1. Introduction. W. Boyce [1] and J. Huneke [4] have shown that if $f$ and $g$ are two commuting continuous self maps of $I$, the closed unit interval $[0,1]$, then $f$ and $g$ need not have a common fixed-point in $I$. However, Boyce [2, Corollary 5] has found that if at least one of the two maps has the additional property that the set of all its iterates (under functional composition) is equicontinuous, then $f$ and $g$ do indeed have a common fixed-point.

This latter result suggests the following problem. Suppose that $F$ is a family of three or more commuting continuous self maps of $I$. Suppose further that, with one possible exception, each $f \in F$ has the property that the set of all its iterates is equicontinuous. It is clear, of course, from Boyce's work that each pair of maps $f, g \in F$ must have a common fixedpoint. But this still leaves open the question of whether $I$ contains a fixedpoint common to all $f \in F$.

In Theorem 3, we resolve this question in the affirmative for all such families $F$, whether finite or infinite. This is obtained by an application of Theorems 1 and 2, which in turn are shown by the use of topological semigroup methods. Much of the technique that is employed in the proof of these results appears in the work of A. L. Shields [6].

Presented in part to the Society, January 23, 1969; received by the editors September 8,1970

AMS 1970 subject classifications. Primary 54H25, 54H15; Secondary 26A18, 54C05, 22A $15,54 \mathrm{C} 30$.

Key nords and phrases. Common fixed-points, equicontinuous mappings, left reversible semigroup, compact topological semigroups, commuting real functions.

${ }^{1}$ This work was supported by a Temple University Faculty Research Award.

- American Mathematical Society 1972 
I am greatly indebted to the referee for his helpful suggestions. In particular, the strengthened forms in which Theorems 1 and 3 appear are due to him.

2. Preliminaries. A semigroup is a set equipped with an associative binary composition called the semigroup product. A semigroup $S$ is left reversible if for every pair of elements $a, b \in S$, there exists a pair $c, d \in S$ such that $a c=b d$. It is evident that every commutative semigroup is left reversible. Let $X$ be a compact Hausdorfi space, and $\mathscr{U}$ the unique uniformity determined by the topology of $X$ (see [5, p. 197]). A family $F$ of self maps of $X$ is equicontinuous if for each $y \in X$ and $U \in \mathbb{U}$, there exists $V \in \mathscr{U}$ such that $(x, y) \in V$ implies $(f x, f y) \in U$ for all $f \in F$. The family $F$ is uniformly equicontinuous if for each $U \in \mathbb{U}$. there exists $V \in \mathscr{U}$ such that $(x, y) \in V$ implies $(f x, f y) \in U$ for all $f \in F$. A family of self maps of a compact Hausdorff space is equicontinuous if and only if the family is uniformly equicontinuous [5, pp. 239-240]. All semigroups of self maps of $X$ that appear in this paper have functional composition as the semigroup product. The semigroup of continuous self maps of $X$, designated by $C(X, X)$ is given the topology of uniform convergence on $X$. If $F \subseteq C(X, X)$, then $F$ denotes the closure of $F$ in $C(X, X)$.

\section{Fixed-point theorems.}

THEOREM 1. Let $X$ be a compact Hausdorff space and let $S$ be an equicontinuous semigroup of self maps of $X$. If $S$ is left reversible, then there is a retract $Y$ of $X$ and a compact subgroup $G$ of $S$ such that $G$, restricted to $Y$, is a group of homeomorphisms of $Y$ onto itself. Further, the elements of $S$ have a common fixed-point $p \in X$ if and only if $p \in Y$ and $p$ is a common fixed-point of $G$.

Proof. Since $C(X, X)$ is given the topology of uniform convergence on $X$, then $C(X, X)$ forms a topological semigroup, that is, a semigroup with a Hausdorff topology in which the semigroup product is jointly continuous (see [3. Example 14.1, p. 269]). But $S$ is an equicontinuous subsemigroup of $C(X . X)$, so its closure $S$ is a compact topological semigroup [3, p. 270], $[5$, p. 232].

We wish to show that $S$ is left reversible. Let $a, b \in \bar{S}$, then there exist nets $\{a(\gamma)\}$ and $\{b(\gamma)\}$ in $S$ such that $a(\gamma) \rightarrow a$ and $b(\gamma) \rightarrow b$. By left reversibility of $S$, there exist nets $\{c(\gamma)\}$ and $\{d(\gamma)\}$ in $S$ for which $a(\gamma) c(\gamma)=b(\gamma) d(\gamma)$. Since $S$ is compact, there exist subnets $\{c(\delta)\}$ and $\{d(\delta)\}$ for which $c(\delta) \rightarrow c$ and $d(\delta) \rightarrow d$ for some $c, d \in S$. Hence

$$
\begin{aligned}
a c & =(\lim a(\delta))(\lim c(\delta))=\lim (a(\delta) c(\delta))=\lim (b(\delta) d(\delta)) \\
& =(\lim b(\delta))(\lim d(\delta))=b d,
\end{aligned}
$$


so $S$ is left reversible. (If $X$ is metrisable, then $C(X, X)$ is also, so the nets in the above argument could be replaced in such a case by sequences. But if $X$ is not first-countable, then neither is $C(X, X)$.)

A right ideal $R$ [left ideal $L$ ] of a semigroup $W$ is a subset of $W^{\prime}$ for which $R W \subseteq R[W L \subseteq L]$. Clearly, if the intersection $R$ of a family of right ideals of a semigroup is nonempty, then $R$ is also a right ideal. But $a S \cap b S \neq \varnothing$ for all $a, b \in \bar{S}$, therefore the compact semigroup $S$ has a unique minimal right ideal $K$, where $K=\bigcap\{a S ; a \in S\}$. However, a compact semigroup $W$ contains minimal left ideals; further $W$ contains a unique minimal right ideal if and only if each minimal left ideal of $W$ is a compact topological group (see [3, Theorem 1, p. 57]). Hence $S$ contains a left ideal $G$ which is also a compact group.

Let $e$ designate the identity element of $G$, and let $Y=e X$. But $e^{2}=e$, where $e \in C(X, X)$, so it follows that $Y$ is a retract of $X$ and that $e y=y$ for all $y \in Y$. Let $f \in G, y \in Y$, and let $g$ be the inverse element of $f$ in the group $G$; then $f y=(e f) y=e(f y) \in Y$, and also $(f g) y=(g f) y=e y=y$. Thus $G$, restricted to $Y$, forms a group $\mathscr{G}$, homomorphic to $G$, of homeomorphisms of $Y$ onto itself. But if an $h \in G$ satisfies $h y=y$ for all $y \in Y$, then

$$
h x=(h e) x=h(e x)=e x
$$

for all $x \in X$, so $h=e$. Hence the restriction map from $G$ onto $\mathscr{G}$ given by $f \rightarrow f \mid Y$ is actually an isomorphism, and (by compactness of $G$ and [5, Theorem 8, p. 141]) is also a homeomorphism when $\mathscr{G}$ is given the topology of uniform convergence on $Y$.

Suppose, now, that $S$ has a common fixed-point $p \in X$. The map from $S$ to $X$ given by $a \rightarrow a p$ is continuous by [5, Theorem 9, p. 227]. so the set $\{a \in S ; a p=p\}$ is closed in $S$, hence equals $S$ since $S$ is dense in $S$. But $G \subseteq S$, therefore $f p=p$ for all $f \in G$, thus $p=e p \in Y$.

Now let $p$ be a common fixed-point of $G$, where $p \in X$. Then $p=e p \in Y$. But $a e \in G$ for all $a \in S$ because $G$ is a left ideal of $S$. Thus for all $a \in S$, we have $a p=a(e p)=(a e) p=p$, which proves Theorem 1 .

It is an open question whether a compact abelian group $G$ of homeomorphisms of a retract $Y$ of a compact convex subset $X$ of a locally convex space has a common fixed-point. (The referee has informed the author that this seems to be unknown, even if $X$ is the $n$-cell.) In any event, the problem of whether an equicontinuous !eft reversible abelian semigroup $S$ of self maps of $X$ has a common fixed-point is equivalent, by Theorem 1 , to this open question. However, something can be said about the case where $X=I$, the closed unit interval. We need a known result [3, p. 333].

LEMma 1. Let $J$ be a closed bounded interval, and let $G$ be a compact group of homeomorphisms of $J$ onto itself. 
(i) If $f \in G$ is order preserving on $J$, then $f=e$, the identity map on $J$.

(ii) G has at most two elements.

Proof. (i) There exists a sequence $\{n(i)\}$ of positive integers such that $\left\{f^{n(i)}\right\}$ converges to $e$ uniformly on $J$ [3, Proposition 1.17, p. 15]. Suppose there exists $x \in J$ such that $f x \neq x$, say $f x<x$. Then $f^{2} x<f x$, and by induction, $f^{n} x \leqq f x<x$ for all positive integers $n$. Hence $\left\{f^{n(i)}\right\}$ cannot converge uniformly to $e$, a contradiction.

(ii) By (i), $e$ is the only order preserving element of $G$. Suppose, $f, g \in G$ are order reversing, then $f^{2}$ and $f g$ are order preserving, so $f^{2}=e=f g$. Thus $f=g$ since $G$ is a group.

The next result is an easy consequence of Theorem 1 and Lemma 1.

THEOREM 2. Let $S$ be a left reversible semigroup of equicontinuous self maps of I, the closed unit interval. If a continuous self map $h$ of I commutes with the elements of $S$, then I contains a common fixed-point of $S$ and $h$.

Proof. (We remark that the identity map on $I$ obviously is such an $h$.) By continuity of the semigroup product on $C(I, I), h$ commutes with the elements of $S$. Let $Y$ be the retract of $I$, and $G$ the compact subgroup of $S$ which were shown to exist in Theorem 1. Then $Y$ is a closed subinterval of $I$, and since $h$ commutes with the idempotent $e \in G$, then $h$ maps $Y$ into itself. By Lemma $1, G$ has at most two elements. If $G=\{e\}$, then any fixed-point $p \in Y$ of $h$ is a fixed-point of $G$, hence by Theorem 1, also of $S$. If $G=\{e, f\}$, then $f$ is sense reversing on $Y$ by Lemma 1 , so $f$ has a unique fixed-point $p \in Y$ which must also be a fixed-point for $h$, since $h$ and $f$ commute. However, $p$ is a fixed-point of $S$ by Theorem 1, which proves Theorem 2.

The next item is a generalization of Boyce's result [2, Corollary 5] for the case of two maps. The family of iteraies of an $f \in C(I, I)$ is the set $\left\{f^{n} ; n=0,1, \cdots\right\}$, where $f^{0}$ denotes the identity map on $I$.

THEOREM 3. Let $F$ be a family of pairwise commuting continuous self maps of I such that for each $f \in F$, with one possible exception, the family of iterates of $f$ is equicontinuous. Then I contains a common fixed-point of $F$.

Proof. Let $h \in F$ be the exceptional map, if $F$ contains one, otherwise let $h \in F$ be arbitrarily chosen. Let $B=\left\{f_{i} ; i=1, \cdots, n\right\}$ be any finite subset of $F-\{h\}$, and let $B_{i}$ be the family of iterates of $f_{i}$. Each family $B_{i}$ is equicontinuous, hence uniformly equicontinuous on $I$. It follows easily that the set $T \subseteq C(I, I)$ given by $T=\left\{a_{1} a_{2} \cdots a_{n} ; a_{i} \in B_{i}\right\}$ is uniformly equicontinuous, hence equicontinuous on $I$. By commutativity, the product of any two elements of $T$ can be expressed as an element of $T$, thus $T$ is a commutative (hence a left reversible) semigroup. However, $h$ 
commutes with the elements of $T$, so $T$ and $h$ have a common fixed-point by Theorem 2 . But $B \subseteq T$, thus the sets $\left\{Z_{f} ; f \in F\right\}$ have the finite intersection property, where $Z_{f}=\{x \in I ; f(x)=x\}$. Therefore $\bigcap\left\{Z_{f} ; f \in F\right\} \neq \varnothing$ by compactness of $I$, which proves Theorem 3 .

\section{REFERENCES}

1. W. M. Boyce, Commuting functions with no common fixed point, Trans. Amer. Math. Soc. 137 (1969), 77-92. MR 38 \#4627.

2. - On $\Gamma$-compact maps on an interval and commutativity, Trans. Amer. Math. Soc. 160 (1971), 87-102.

3. K. H. Hofmann and P. S. Mostert, Elements of compact semigroups, Merrill, Columbus, Ohio, 1966. MR 35 \#285.

4. J. P. Huneke, On common fixed points of commuting continuous functions on an interval, Trans. Amer. Math. Soc. 139 (1969), 371-381. MR 38 \#6005.

5. J. L. Kelley, General topology, Van Nostrand, Princeton, N.J., 1955. MR 16, 1136.

6. A. L. Shields, On fixed points of commuting analytic functions, Proc. Amer. Math. Soc. 15 (1964), 703-706. MR 29 \#2790.

Department of Mathematics, Temple University, Philadelphia, Pennsylvania 19122 